Proceedings of the 26th Annual International Conference of the IEEE EMBS

San Francisco, CA, USA • September 1-5, 2004

\title{
BOOTSTRAP-BASED STATISTICAL THRESHOLDING FOR MEG SOURCE RECONSTRUCTION IMAGES
}

\author{
Kensuke Sekihara ${ }^{1}$, Maneesh Sahani ${ }^{2}$, Srikantan S. Nagarajan ${ }^{3}$ \\ ${ }^{1}$ Tokyo Metropolitan Institute of Technology, Tokyo, Japan \\ ${ }^{2}$ Keck Center for Integrated Neuroscience, University of California, San Francisco, CA, USA \\ ${ }^{3}$ Department of Radiology, University of California, San Francisco, CA, USA
}

\begin{abstract}
This paper proposes a bootstrap-based statistical method for extracting target source activities from MEG/EEG source reconstruction results. The method requires measurements in a control condition, which contains only non-target source activities. The method derives, at each pixel location, an empirical probability distribution of the non-target source activity using bootstrapped reconstruction obtained from the control period. The statistical threshold that can extract the target source activities is derived based on the empirical distributions obtained from all pixel locations. Here, the multiple comparison problem is taken into account by using two step procedure: studentizing these empirical distributions and deriving an empirical distribution of the maximum pseudo $T$ value at each pixel location. The results of numerical experiments are presented to demonstrate the method's effectiveness.
\end{abstract}

\section{Keywords - Bootstrap method, MEG, Source reconstruction, Statistical significance test}

\section{INTRODUCTION}

Neuromagnetic imaging can visualize neural activities with a fine time resolution of milli-second order, and provide functional information about brain dynamics. One major problem here is that measured MEG signal generally contains not only magnetic fields associated with the signal sources of interest but also contains interference magnetic fields generated from non-target source activities; such nontarget source activities include brain spontaneous activities or some evoked activities that are not the interest of the current investigation. These non-target activities generally overlap with the target source activities in the source reconstruction, and they often make interpreting the reconstruction results difficult.

In most studies using positron emission tomography or functional magnetic resonance imaging, the experiments are carefully designed to extract only the target activities. A common example for such experimental designs contains two kinds of stimuli: a task stimulus and a control stimulus. The task stimulus generally elicits the target cortical activities as well as other activities associated with the target activities. The control stimulus is designed to elicit only these associated activities. Then, by calculating the statistical subtraction between the images measured with the two kinds of stimulus, the target activities can be extracted.

This paper proposes a bootstrapped-based method for implementing this statistical subtraction between task and control measurements. The method is applicable to results of source reconstruction from evoked MEG/EEG measurements. This method assumes neural activities to be quasi stochastic, and applies the bootstrap method to derive an empirical probability distribution of these activities using the source reconstruction in the control period. This empirical distribution is then used for deriving the statistical threshold; the thresholding can extract the target activities that exist only in the task measurements by eliminating other non-target activities. In this paper, we explain the proposed statistical thresholding method with spatial filter source reconstruction [1]. This is because the formulation of the spatial filter is relatively simple and it has been successfully applied to MEG source analysis. However, the applicability of the proposed method is not limited to the spatial filter formulation and it can be used with any types of source reconstruction methods

\section{SPATIAL FILTER FORMULATION AND PARAMETRIC STATISTICAL SIGNIFICANCE EVALUATION}

We define the magnetic field measured by the $m$ th detector coil at time $t$ as $b_{m}(t)$, and a column vector $\boldsymbol{b}(t)=$ $\left[b_{1}(t), b_{2}(t), \ldots, b_{M}(t)\right]^{T}$ as a set of measured data where $M$ is the total number of sensor coils and superscript $T$ indicates the matrix transpose. The spatial location is represented by a three-dimensional vector $\boldsymbol{r}: \boldsymbol{r}=(x, y, z)$. The magnitude of the source moment is denoted $s(\boldsymbol{r}, t)$. Spatial filter techniques estimate the source current density by 
applying a simple linear operation to the measured data, i.e.,

$$
\widehat{s}(\boldsymbol{r}, t)=\boldsymbol{w}^{T}(\boldsymbol{r}) \boldsymbol{b}(t)=\sum_{m=1}^{M} w_{m}(\boldsymbol{r}) b_{m}(t),
$$

where $\widehat{s}(\boldsymbol{r}, t)$ is the estimated source magnitude. The column vector $\boldsymbol{w}(\boldsymbol{r})$ expresses a set of the filter weights, which characterizes the property of the spatial filter. Various types of spatial filter techniques have been proposed and applied to the MEG/EEG source reconstruction problems. Some of them are found in [2][3][4][5].

The evaluation of the statistical significance of the spatial filter outputs has been conventionally performed using the parametric statistics [2][5]. The basic assumption for such parametric method is that the measurement consists of deterministic signal and Gaussian noise, i.e.,

$$
\boldsymbol{b}(t)=\boldsymbol{b}_{I}(t)+\boldsymbol{n}(t)
$$

where $\boldsymbol{b}_{I}(t)$ is the signal magnetic field of interest, which is generated from brain sources that are the target of current investigation. In Eq. (2), $\boldsymbol{n}(t)$ is the noise vector and each element of $\boldsymbol{n}(t)$ is assumed to follow $\mathcal{N}\left(0, \sigma_{0}^{2}\right)$, which indicates the Gaussian distribution with zero mean and the variance of $\sigma_{0}^{2}$. Since these Gaussian processes are assumed to be uncorrelated between different sensor recordings, the spatial filter outputs $\widehat{s}(\boldsymbol{r}, t)$ expressed as

$$
\widehat{s}(\boldsymbol{r}, t)=\boldsymbol{w}^{T}(\boldsymbol{r}) \boldsymbol{b}(t)=\boldsymbol{w}^{T}(\boldsymbol{r}) \boldsymbol{b}_{I}(t)+\boldsymbol{w}^{T}(\boldsymbol{r}) \boldsymbol{n}(t),
$$

follows

$$
\mathcal{N}\left(\boldsymbol{w}^{T}(\boldsymbol{r}) \boldsymbol{b}_{I}(t), \sigma_{0}^{2}\|\boldsymbol{w}(\boldsymbol{r})\|^{2}\right) .
$$

Thus, using this property, the statistical evaluation can be performed by testing the null hypothesis $\left(\boldsymbol{b}_{I}(t)=0\right)$ at each pixel location.

\section{PROPOSED NONPARAMETRIC STATISTICAL SIGNIFICANCE EVALUATION}

The signal and the noise model expressed in Eq. (2) is, in general, insufficient to express real-world measurements, and the measured data should be expressed as

$$
\boldsymbol{b}(t)=\boldsymbol{b}_{I}(t)+\boldsymbol{b}_{\xi}(t)+\boldsymbol{n}(t),
$$

where $\boldsymbol{b}_{\xi}(t)$ is the magnetic field generated from sources other than the signal sources, such as brain spontaneous activities or some evoked activities that are not the target of current investigation. This $\boldsymbol{b}_{\xi}(t)$ is often referred to as the brain noise. The parametric modeling cannot efficiently take this brain noise into account, because the Gaussianity assumption does not hold for $\boldsymbol{b}_{\xi}(t)$. Here, we propose a bootstrap-based nonparametric method that can take such brain noise into consideration. The spatial filter outputs obtained from $\boldsymbol{b}(t)$ is expressed as

$$
\widehat{s}(\boldsymbol{r}, t)=\widehat{s}_{I}(\boldsymbol{r}, t)+\widehat{s}_{c}(\boldsymbol{r}, t),
$$

where

$$
\begin{aligned}
& \widehat{s}_{I}(\boldsymbol{r}, t)=\boldsymbol{w}^{T}(\boldsymbol{r}) \boldsymbol{b}_{I}(t) \\
& \widehat{s}_{c}(\boldsymbol{r}, t)=\boldsymbol{w}^{T}(\boldsymbol{r})\left(\boldsymbol{b}_{\xi}(t)+\boldsymbol{n}(t)\right)
\end{aligned}
$$

The key assumption in the proposed method is that the control measurement that can provide $\boldsymbol{b}_{c}(t)=\boldsymbol{b}_{\xi}(t)+\boldsymbol{n}(t)$ is available. Using this control measurement, the proposed method first derive an empirical distribution of $\widehat{s}_{c}(\boldsymbol{r}, t)$, and with this empirical distribution, the method evaluates the statistical significance of $\widehat{s}(\boldsymbol{r}, t)$ under the null hypothesis that $\widehat{s}_{I}(\boldsymbol{r}, t)=0$. The proposed procedures are described as follows:

(i) Empirical distribution formation In evoked measurements, the magnetic field is generally obtained by averaging raw-epoch measurements. We denote such raw epochs for the control measurements $\boldsymbol{b}_{c}(t)$ as $\left\{\boldsymbol{e}_{1}(t), \ldots, \boldsymbol{e}_{K}(t)\right\}$ where $K$ is the number of raw epochs, i.e.,

$$
\boldsymbol{b}_{c}(t)=1 / K \sum_{j=1}^{K} \boldsymbol{e}_{j}(t) .
$$

We calculate the bootstrapped control measurements $\boldsymbol{b}_{c}^{\beta}(t)$ such that

$$
\boldsymbol{b}_{c}^{\beta}(t)=1 / K \sum_{j=1}^{K} \boldsymbol{e}_{j}^{*}(t),
$$

where $\left\{\boldsymbol{e}_{1}^{*}(t), \ldots, \boldsymbol{e}_{K}^{*}(t)\right\}$ are the re-sampled epochs, each of which is drawn randomly from $\left\{\boldsymbol{e}_{1}(t), \ldots, \boldsymbol{e}_{K}(t)\right\}$ with replacements. The assumption here is that each epoch is a one realization of a stochastic process with unknown distribution. In Eq. (9), $\beta$ is the bootstrap index such that $\beta=1, \ldots, K_{B}$ where $K_{B}$ indicates the total number of bootstrap samples. We next calculate $\widehat{s}_{c}^{\beta}(\boldsymbol{r}, t)$ by applying the spatial filter to $\boldsymbol{b}_{c}^{\beta}(t)$, i.e., $\widehat{s}_{c}^{\beta}(\boldsymbol{r}, t)=\boldsymbol{w}^{T}(\boldsymbol{r}) \boldsymbol{b}_{c}^{\beta}(t)$, and obtain

$$
p_{c}^{\beta}(\boldsymbol{r})=\sqrt{\left\langle\widehat{s}_{c}^{\beta}(\boldsymbol{r}, t)^{2}\right\rangle},
$$

where $\langle\cdot\rangle$ indicate the time average over the control period. We then calculate $\widehat{F}(x)$, which is the empirical distribution of $p_{c}^{\beta}(\boldsymbol{r})$, such that $\widehat{F}(x)=\sharp\left\{p_{c}^{\beta}(\boldsymbol{r}) \leq x\right\} / K_{B}$ where $\sharp\left\{p_{c}^{\beta}(\boldsymbol{r}) \leq x\right\}$ indicates the number of $p_{c}^{\beta}(\boldsymbol{r})$ which are less than or equal to $x$. At all pixel locations, this procedure is repeated and the empirical distribution is calculated. Since $\widehat{F}(x)$ is obtained at each pixel location $\boldsymbol{r}, \widehat{F}(x)$ is rewritten as $\widehat{F}(x \mid \boldsymbol{r})$ in the following explanation.

(ii) Statistical thresholding without multiple comparisons Using $\widehat{F}(x \mid \boldsymbol{r})$, we could obtain the statistical threshold at $\boldsymbol{r}, \Sigma(\boldsymbol{r})$, such that $\Sigma(\boldsymbol{r})=\widehat{F}^{-1}(1-\alpha \mid \boldsymbol{r})$ where $1-\alpha$ 
is the confidence level such as $1-\alpha=0.95$. In practice, the inverse of the empirical distribution can be calculated by first sorting $p_{c}^{\beta}(\boldsymbol{r})$ in the increasing order with respect to $\beta$,

$$
p_{c}^{(1)}(\boldsymbol{r}) \leq p_{c}^{(2)}(\boldsymbol{r}) \leq \cdots \leq p_{c}^{\left(K_{B}\right)}(\boldsymbol{r}),
$$

where $p_{c}^{(j)}(\boldsymbol{r})$ is the $j$ th smallest item among $p_{c}^{\beta}(\boldsymbol{r})$. Then, we choose $p_{c}^{(q)}(\boldsymbol{r})$ as $\Sigma(\boldsymbol{r})$ where $q=\left[(1-\alpha) K_{B}\right]$ and $[\cdot]$ indicates the maximum integer that does not exceed the value in the parenthesis. However, the statistical threshold obtained in this manner does not take the multiple comparisons into consideration, and instead of implementing the above-mentioned procedure, the following procedure should be performed.

(iii) Statistical thresholding with multiple comparisons The proposed method uses the maximum statistics to incorporate the multiple comparison problems; the use of the maximum statistics has been studied in [6]. To utilize the maximum statistics, we first studentize the empirical distribution of $p_{c}^{\beta}(\boldsymbol{r})$ by calculating $T_{\beta}(\boldsymbol{r})$ such that

$$
T_{\beta}(\boldsymbol{r})=\frac{p_{c}^{\beta}(\boldsymbol{r})-\left\langle p_{c}^{\beta}(\boldsymbol{r})\right\rangle_{\beta}}{\widehat{\sigma}(\boldsymbol{r})} .
$$

Here,

$$
\widehat{\sigma}^{2}(\boldsymbol{r})=\left\langle p_{c}^{\beta}(\boldsymbol{r})^{2}\right\rangle_{\beta}-\left\langle p_{c}^{\beta}(\boldsymbol{r})\right\rangle_{\beta}^{2},
$$

and $\langle\cdot\rangle_{\beta}$ indicates the average over the bootstrap samples.

We then calculate $T_{\max }(\boldsymbol{r})$, which is the maximum $T_{\beta}(\boldsymbol{r})$ value. The maximum $T_{\beta}$ value at the $i$ th pixel location is denoted $T_{\text {max }}^{i}$ where $i=1, \ldots, K_{N}$ and $K_{N}$ indicates the total number of pixels. We next obtain the empirical distribution of $T_{\max }^{i}, \widehat{H}(x)$, such that $\widehat{H}(x)=\sharp\left\{T_{\max }^{i} \leq\right.$ $x\} / K_{N}$ where $\sharp\left\{T_{\text {max }}^{i} \leq x\right\}$ is the number of $T_{\text {max }}^{i}$ values which are less than or equal to $x$. We can obtain the threshold for the $(1-\alpha)$ confidence level, $T^{\text {th }}$, such that $T^{t h}=\widehat{H}^{-1}(1-\alpha)$. The inverse of this empirical distribution can be calculated by first sorting $T_{\text {max }}^{i}$ in the increasing order

$$
T_{\max }^{(1)} \leq T_{\max }^{(2)} \leq \cdots \leq T_{\max }^{\left(K_{N}\right)},
$$

and choose $T_{\max }^{(p)}$ as $T^{t h}$ where $p=\left[(1-\alpha) K_{N}\right]$. We finally obtain the statistical threshold for the spatial-filter reconstruction, $\Sigma(\boldsymbol{r})$, by using

$$
\Sigma(\boldsymbol{r})=T^{t h} \widehat{\sigma}(\boldsymbol{r})+\left\langle p_{c}^{\beta}(\boldsymbol{r})\right\rangle_{\beta} .
$$

We evaluate the statistical significance of the spatial filter outputs by comparing the outputs $|\widehat{s}(\boldsymbol{r}, t)|$ with $\Sigma(\boldsymbol{r})$, and when $|\widehat{s}(\boldsymbol{r}, t)| \geq \Sigma(\boldsymbol{r})$, the outputs $\widehat{s}(\boldsymbol{r}, t)$ is considered to be statistically significant.

\section{NUMERICAL EXPERIMENTS}

We conducted numerical experiments to show the effectiveness of the proposed statistical thresholding. We use a sensor alignment of the 37-sensor array from Magnes ${ }^{\mathrm{TM}}$ (4D
Neuroimaging Inc., San Diego) neuromagnetometer. Three signal sources were assumed to exist on a single plane $(x=$ $0 \mathrm{~cm}$ ). The source-sensor configuration and the coordinate system are illustrated in Fig. 1. The simulated magnetic

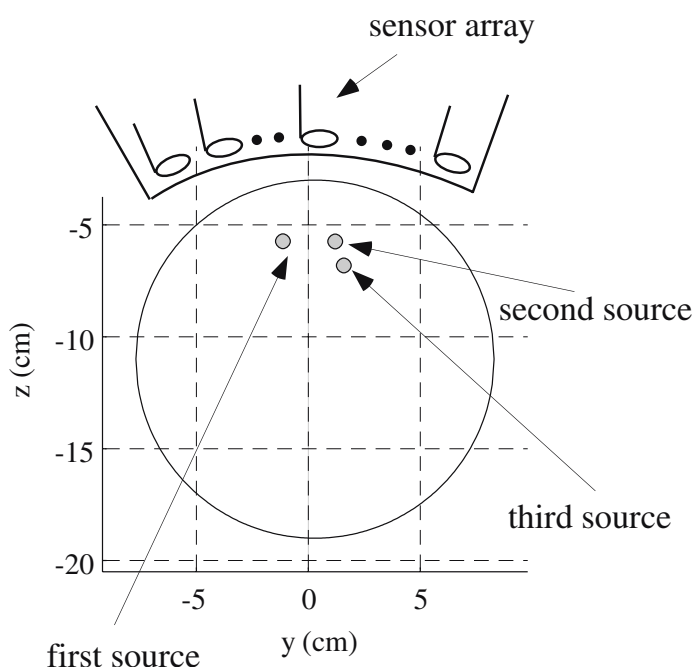

Fig. 1. The coordinate system and source-sensor configuration used in the numerical experiments. The circle indicate the projection of the sphere used for the forward calculation.

field were calculated at $400 \mathrm{~ms}$ prestimulus and $400 \mathrm{~ms}$ post stimulus time window with $1 \mathrm{~ms}$ sample. Here, nearly orthogonal three time courses shown in Fig. 2 were assigned to the three sources. In this experiments, the prestimulus period is considered as the control period. The first and the second sources is considered as the signal sources of interest and the third sources as the control sources that are active both the post and pre stimulus periods. Spontaneous MEG measured using the same sensor array was added to this simulated signal-magnetic field to create a simulated raw-epoch data. One hundred raw-epochs were generated in this manner, and averaged to create the final simulated evoked recordings. The resultant simulated evoked recordings are also shown in Fig. 2.

The eigenspace-projected adaptive spatial filter [4] was applied to this averaged recordings. The data between 0 to $400 \mathrm{~ms}$ was used for calculating the covariance matrix and the weight vector of the spatial filter was obtained with this covariance matrix. The spatial filter was applied to both the pre and post stimulus $400 \mathrm{~ms}$ time windows to give two sets of the source power reconstruction results, $\sqrt{\left\langle\widehat{\left.\hat{s}(\boldsymbol{r}, t)^{2}\right\rangle_{\text {pre }}}\right.}$ and $\sqrt{\left\langle\hat{s}(\boldsymbol{r}, t)^{2}\right\rangle_{\text {post }}}$. These results are shown in Fig. 3, indicating that all three sources are active in the post stimulus period but only the third source is active in the prestimulus period. Then, the proposed method was applied the post stimulus reconstruction $\sqrt{\left\langle\widehat{s}(\boldsymbol{r}, t)^{2}\right\rangle_{\text {post }}}$. The results of thresholding $\sqrt{\left\langle\widehat{s}(\boldsymbol{r}, t)^{2}\right\rangle_{\text {post }}}$ with $95 \%$ confidence level are shown in Fig. 4. The results indicate that the third source 
is eliminated from the post stimulus reconstruction, verifying the effectiveness of the proposed method.

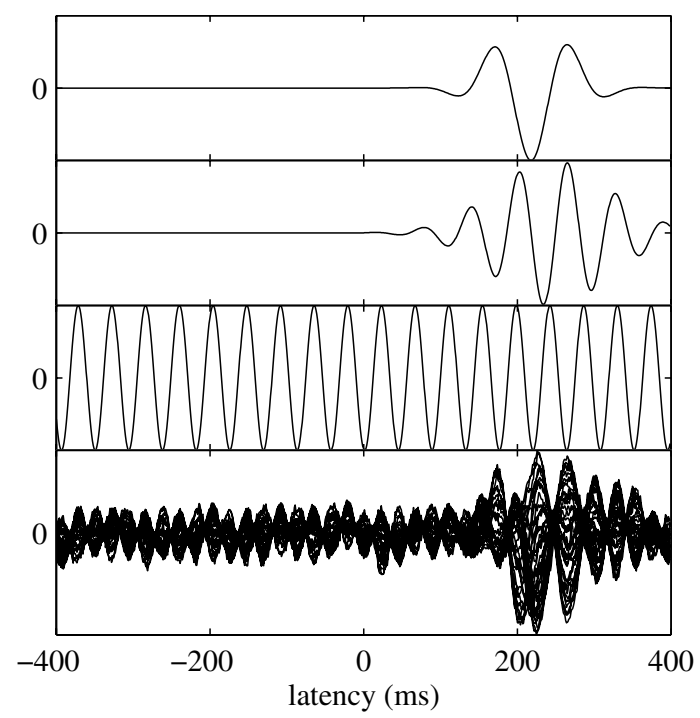

Fig. 2. Time courses assigned to the three sources and simulated evoked recordings (bottom).

\section{REFERENCES}

[1] K. Sekihara and S. S. Nagarajan, "Neuromagnetic source reconstruction and inverse modeling," in Modeling and Imaging of Bioelectric Activity - Principles and Applications, Kluwer Academic/Plenum Publishers, in press.

[2] S. E. Robinson and J. Vrba, "Functional neuroimaging by synthetic aperture magnetometry (SAM)," in Recent Advances in Biomagnetism, Sendai, pp., 302-305, Tohoku University Press.

[3] B. D. van Veen and W. van Drongelen and M. Yuchtman and A. Suzuki, "Localization of brain electrical activity via linearly constrained minimum variance spatial filtering," IEEE Trans. Biomed. Eng., vol. 44, pp. 867-880, 1997.

[4] K. Sekihara and S. S. Nagarajan and D. Poeppel and A. Marantz and Y. Miyashita, "Application of an MEG eigenspace beamformer to reconstructing spatiotemporal activities of neural sources," Human Brain Mapping, vol. 15, pp. 199-215, 2002.

[5] A. M. Dale and A. K. Liu and B. R. Fischl and R. L. Buckner and J. W. Belliveau and J. D. Lewine and E. Halgren, "Dynamic statistical parametric mapping: Com-
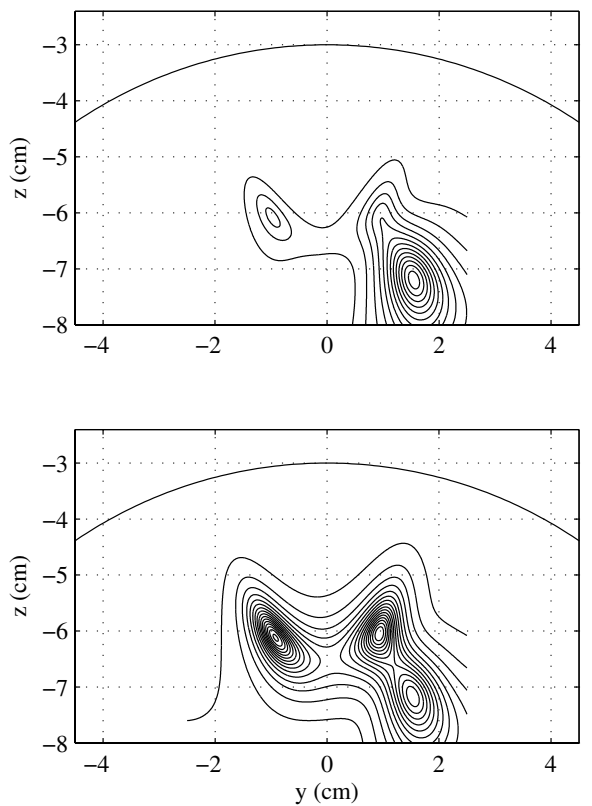

Fig. 3. Source reconstruction results for the prestimulus pe$\operatorname{riod} \sqrt{\left\langle\hat{s}(\boldsymbol{r}, t)^{2}\right\rangle_{\text {pre }}}$ (upper) and for the poststimulus period $\sqrt{\left\langle\widehat{s}(\boldsymbol{r}, t)^{2}\right\rangle_{\text {post }}}$ (lower)

bining fMRI and MEG for high-resolution imaging of cortical activity," Neuron, vol. 26, pp. 55-67, 2000.

[6] D. Pantazis and T. E. Nichols and S. Baillet and R. M. Leahy, "Spatiotemporal localization of significant activation in MEG using permutation tests," in Conference Report for the Information Processing in Medical Imaging 2003, Ambleside, UK, pp., 99-999.

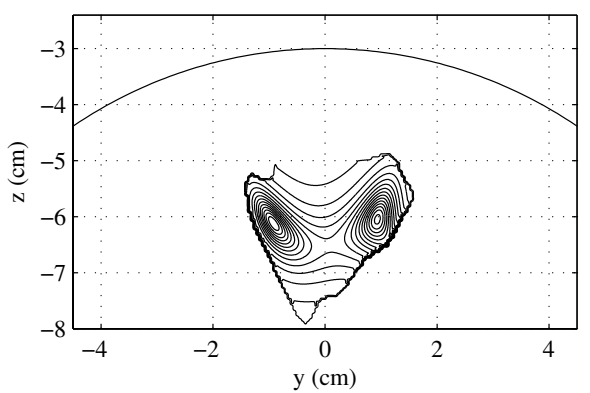

Fig. 4. Source reconstruction results $\sqrt{\left\langle\widehat{s}(\boldsymbol{r}, t)^{2}\right\rangle_{\text {post }}}$ thresholded by the proposed method. 